\title{
Effects of electronic and lattice polarization on the band structure of delafossite transparent conductive oxides
}

\author{
Julien Vidal, ${ }^{1,2,3}$ Fabio Trani, ${ }^{4,3}$ Fabien Bruneval,, 3 \\ Miguel A. L. Marques, ${ }^{4,3}$ and Silvana Botti ${ }^{2,4,3}$ \\ ${ }^{1}$ Institute for Research and Development of Photovoltaic Energy (IRDEP), \\ UMR 7174 CNRS/EDF/ENSCP, 6 quai Watier, 78401 Chatou, France \\ ${ }^{2}$ Laboratoire des Solides Irradiés, École Polytechnique, \\ CNRS, CEA-DSM, 91128 Palaiseau, France \\ ${ }^{3}$ European Theoretical Spectroscopy Facility (ETSF) \\ ${ }^{4}$ LPMCN, Université Claude Bernard Lyon I and CNRS, 69622 Villeurbanne, France \\ ${ }^{5}$ CEA, DEN, Service de Recherches de Métallurgie Physique, F-91191 Gif-sur-Yvette, France

\begin{abstract}
We use hybrid functionals and restricted self-consistent $G W$, state-of-the-art theoretical approaches for quasiparticle band structures, to study the electronic states of delafossite $\mathrm{Cu}(\mathrm{Al}, \mathrm{In}) \mathrm{O}_{2}$, the first p-type and bipolar transparent conductive oxides. We show that self-consistent $G W$ gives remarkably wider band gaps than all the other approaches used so far. Accounting for polaronic effects in the $G W$ scheme we recover a very nice agreement with experiments. Furthermore, the modifications with respect to the Kohn-Sham bands are strongly $\boldsymbol{k}$-dependent, which makes questionable the common practice of using a scissor operator. Finally, our results support the view that the low energy structures found in optical experiments, and initially attributed to an indirect transition, are due to intrinsic defects in the samples.
\end{abstract}

PACS numbers: 71.20.-b 71.45.Gm 78.20.-e 71.15.Qe 71.35.Cc 
Many high-technology devices, such as flat panel displays, touch screens, or even thinfilm solar cells, require the use of thin transparent contacts. These contacts are usually built from insulating oxides that, for a certain range of doping, become conductive while retaining transparency in the visible spectrum. The most common examples of these socalled transparent conductive oxides (TCOs) are electron (n-) doped $\mathrm{SnO}_{2}, \mathrm{In}_{2} \mathrm{O}_{3}$, and $\mathrm{ZnO}$. Hole (p-)doping of wide gap semiconductors was for long time very hard to obtain [1]. It is therefore not surprising that the discovery of p-doping in $\mathrm{CuAlO}_{2}$ thin films with a carrier mobility of about $10 \mathrm{~cm}^{2} /(\mathrm{Vs})$ attracted great interest [2]. Other members of the delafossite family, like $\mathrm{CuGaO}_{2}$ [3] and $\mathrm{CuInO}_{2}$ [4], were discovered shortly after. The latter compound is particularly interesting as it exhibits bipolar (n- and p-type) conductivity by doping with appropriate impurities and tuning the film-deposition conditions [4]. This opens the way to the development of transparent $\mathrm{p}-\mathrm{n}$ junctions, and therefore fully transparent optoelectronic devices, functional windows and stacked solar cells with improved efficiency.

$\mathrm{CuAlO}_{2}$ is by far the most studied system of the family of delafossite TCOs. However, there is still no agreement either on the origin of the p-type conductivity, or on the electronic bands of the pure crystal. Measurements of the direct optical band gap $\left(E_{\mathrm{g}}^{\mathrm{dir}}\right)$ of $\mathrm{CuAlO}_{2}$ fall in the range from 2.9 to $3.9 \mathrm{eV}$ [2, 5 - 8], with most values in the interval $3.4-3.7 \mathrm{eV}$. These experiments also yield a large dispersion of indirect gaps $\left(E_{\mathrm{g}}^{\text {ind }}\right)$, from 1.65 to $2.1 \mathrm{eV}$, with one experiment measuring $2.99 \mathrm{eV}$ [8]. Unfortunately, there is only one photoemission experiment [5] that yields $3.5 \mathrm{eV}$ for the quasiparticle band gap. Note that the optical and quasiparticle gaps differ by the exciton binding energy. Concerning $\mathrm{CuInO}_{2}$, optical experiments measured $E_{\mathrm{g}}^{\mathrm{dir}}$ between 3.9 and $4.45 \mathrm{eV}$ [4, 9, 10], with only one estimation of $E_{\mathrm{g}}^{\text {ind }}$ at $1.44 \mathrm{eV}[10]$.

From the theoretical perspective, the situation is also quite complex, even if the full $\mathrm{Cu}$ $3 d$ shell should exclude the strongly correlated electron regime. These materials are usually studied within density functional theory (DFT), using the standard local density (LDA) or generalized gradient approximations (GGA). However, it is well known that the Kohn-Sham band structures systematically underestimate the band gaps. For similar compounds, like $\mathrm{Cu}_{2} \mathrm{O}$ and $\mathrm{CuIn}(\mathrm{S}, \mathrm{Se})_{2}$, Kohn-Sham LDA calculations lead to unreasonable band structures, in particular due to the misrepresentation of the hybridization between the $d$ electrons of the metal and $p$ electrons of the anion [11, 12]. To overcome this situation, hybrid functionals have been recently proposed, with very promising results [13], especially for materials with 
small and intermediate band gaps [14, 15]. Other approaches include $\mathrm{LDA}+U$, that tries to improve the description of correlation through the introduction of a mean-field Hubbard-like term. This method has been quite successful in the study of strongly correlated systems, but it relies on a parameter $U$, that is often adjusted to experiments.

Arguably the most reliable and used ab initio technique to obtain quasi-particle band structures is the many-body $G W$ approach [16]. The common practice within this framework is to start from a DFT calculation, and evaluate perturbatively the $G W$ energy corrections to the band structure. This procedure, which we will refer to as $G_{0} W_{0}$, is justified when the departure wave functions and band structure are already close to the quasiparticle ones. This is indeed the case in many systems, explaining why $G_{0} W_{0}$ has been extremely successful in describing electron addition and removal energies for metals, semiconductors and insulators [17]. However, it has been recently shown that $G_{0} W_{0}$ fails for many transition metal oxides [11, 18].

To solve this problem one can perform restricted self-consistent (sc) GW [19]. This technique has the advantage of being independent of the starting point at the price of large computational complexity. Fortunately, there is an alternative procedure that yields wavefunctions that are extremely close to those obtained in a full sc- $G W$ calculation, namely sc-COHSEX as explained in Ref. [20]. The dynamical effects that are absent in COHSEX calculations can then be accounted for by performing a final perturbative $G W$ step. This method, that we will refer to as sc- $G W$, has been applied to many oxide compounds, yielding excellent results for the band gaps and the quasiparticle band structure [11, 12, 18, 20].

Note that these theoretical techniques yield quasiparticle bands, and not optical gaps. To evaluate these latter quantities one mostly resorts to the solution of the Bethe-Salpeter equation. For the delafossite structures there is one such calculation starting from a GGA $+U$ band structure [21]. It yields for $\mathrm{CuAlO}_{2}$ a very large exciton binding energy of about $0.5 \mathrm{eV}$ for the first direct transition. The choice of $U$ was found to have strong consequences on the width of the band gap, but it did not affect significantly the exciton binding energy. We can thus assume that $0.5 \mathrm{eV}$ is a reasonable estimate.

In the following, we present calculations of the band structures of $\mathrm{CuAlO}_{2}$ and $\mathrm{CuInO}_{2}$ using some of the most accurate theoretical tools available in the community. These include the standard LDA, hybrid functionals (namely B3LYP [22] and two flavors of Heyd-ScuseriaErnzerhof, HSE03 and HSE06 [23]), LDA $+U, G_{0} W_{0}$ and sc- $G W$. As discussed above, we 


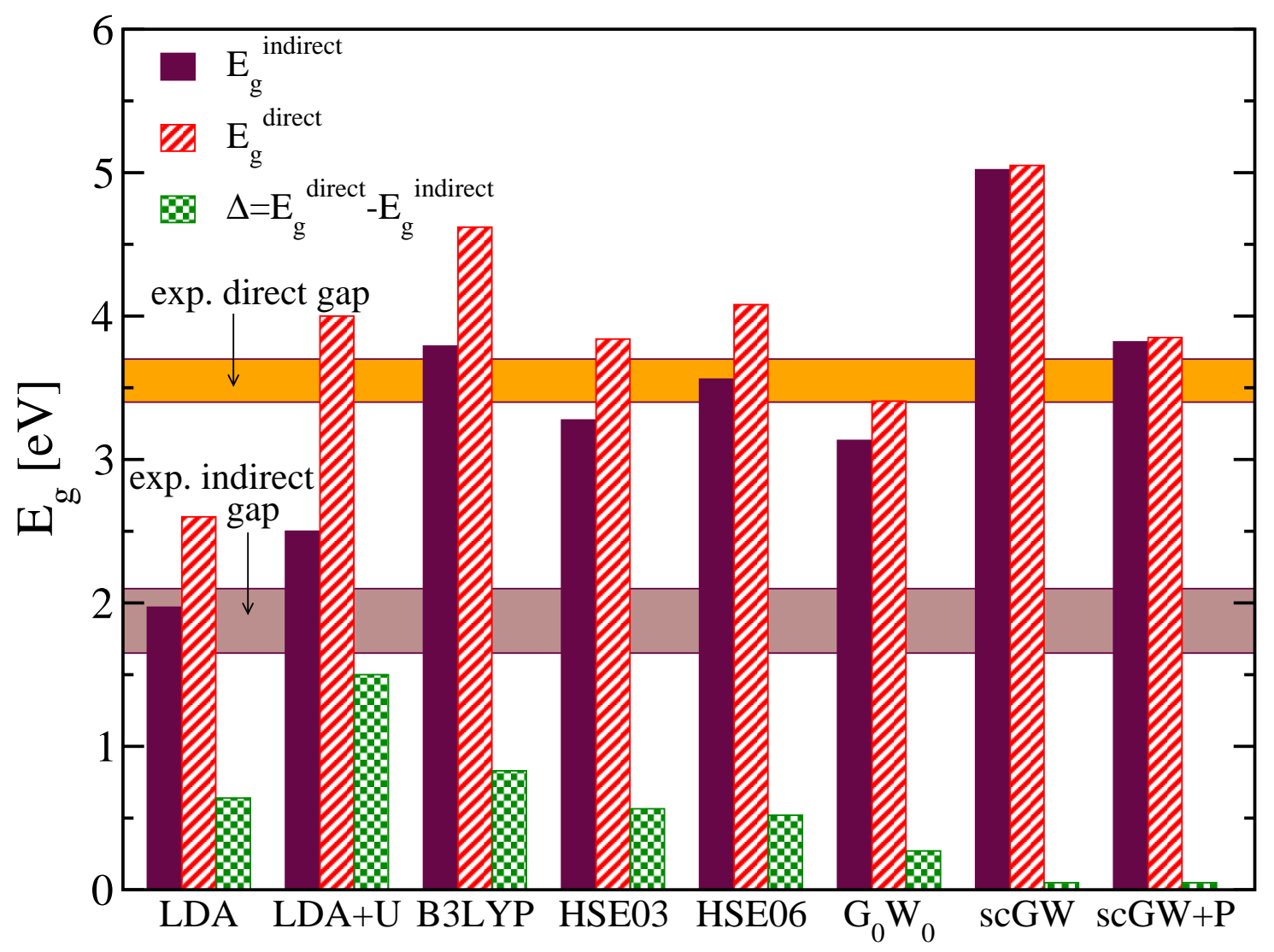

FIG. 1: (Color online) Band gaps of $\mathrm{CuAlO}_{2}$ using: LDA, LDA $+U$, hybrid, $G_{0} W_{0}$, sc- $G W$, and sc- $G W$ including model polaronic corrections. The horizontal zones contain data extracted from various optical experiments (see text).

expect sc- $G W$ to be the most accurate $a b$ initio approach. When the comparison was possible, we found our results in excellent agreement with previous calculations (Refs. [5, 7, 8, 24 26] for LDA, Ref. [26] for B3LYP, and Ref. [27] for GGA+U).

The hybrid and LDA $+U$ calculations were performed with VASP [28] and ABINIT [29] respectively, using the PAW formalism and an energy cutoff of $44 \mathrm{Ha}$. The parameter $U$ was set to $8 \mathrm{eV}$ as in Ref. [21]. Our $G W$ calculations were performed with ABINIT, starting from LDA band structures and using norm-conserving pseudopotentials with semicore states (3s and $3 \mathrm{p}$ for $\mathrm{Cu}$ and $4 \mathrm{~s}$ and $4 \mathrm{p}$ for In) included in the valence. The energy cutoff was $120 \mathrm{Ha}$ for the ground state calculation, and the $\mathbf{k}$-point grid was a $4 \times 4 \times 4$ Monkhorst-Pack. As the experimental and LDA relaxed geometries are very close (within 1\%), and the small contraction of the lattice in LDA has a negligible effect on band structures $(\leq 0.05 \mathrm{eV})$, we employed experimental lattice parameters [26]. Note that it was absolutely essential to use 

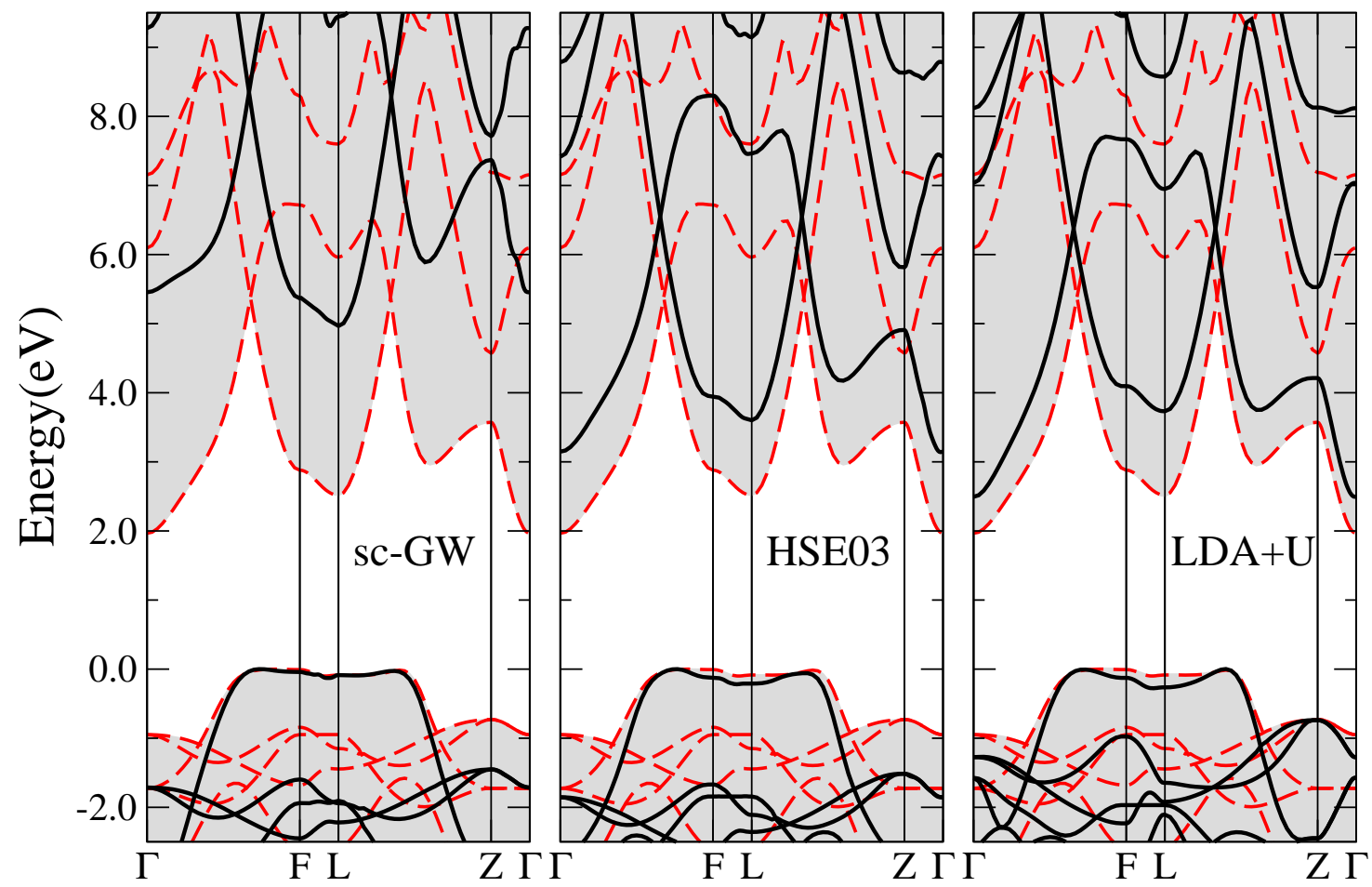

FIG. 2: (Color online) Band structures for $\mathrm{CuAlO}_{2}$ : comparison of LDA (red dashed lines) with sc- $G W$ (left panel), HSE03 (central panel), and LDA $+U$ (right panel).

the method of Ref. [30], due to the extremely slow convergence with respect to the number of empty states.

In Figs. 1 and 2 we show direct and indirect photoemission gaps and the band structures of $\mathrm{CuAlO}_{2}$ obtained using different theoretical approaches. The minimum $E_{\mathrm{g}}^{\mathrm{dir}}$ of $\mathrm{CuAlO}_{2}$ is always found at L, where the dipole transition between the band edge states is allowed [25]. All calculations, except sc- $G W$, give a fundamental $E_{\mathrm{g}}^{\text {ind }}$ between the conduction band minimum at $\Gamma$ and the valence band maximum along the $\Gamma-F$ line. The experimental data for optical gaps are also presented with an error bar that reflects the dispersion of the most likely values found in literature. LDA exhibits, as expected, the smallest gaps. Basically every approach beyond it opens up the gap by different amounts and modifies the band dispersions. The direct and indirect gaps have similar behaviors in the different theories, and both increase when going from $\mathrm{LDA}<G_{0} W_{0}<\mathrm{HSE} 03<\mathrm{HSE} 06<\mathrm{B} 3 \mathrm{LYP}<$ sc- $G W$. On the other hand, the difference $E_{\mathrm{g}}^{\mathrm{dir}}-E_{\mathrm{g}}^{\mathrm{ind}}$ seems to decrease with the sophistication of the method, reaching nearly zero for the sc- $G W$ calculation. This is a consequence of the drastic change of the conduction band dispersion, which displaces the conduction minimum from $\Gamma$ 


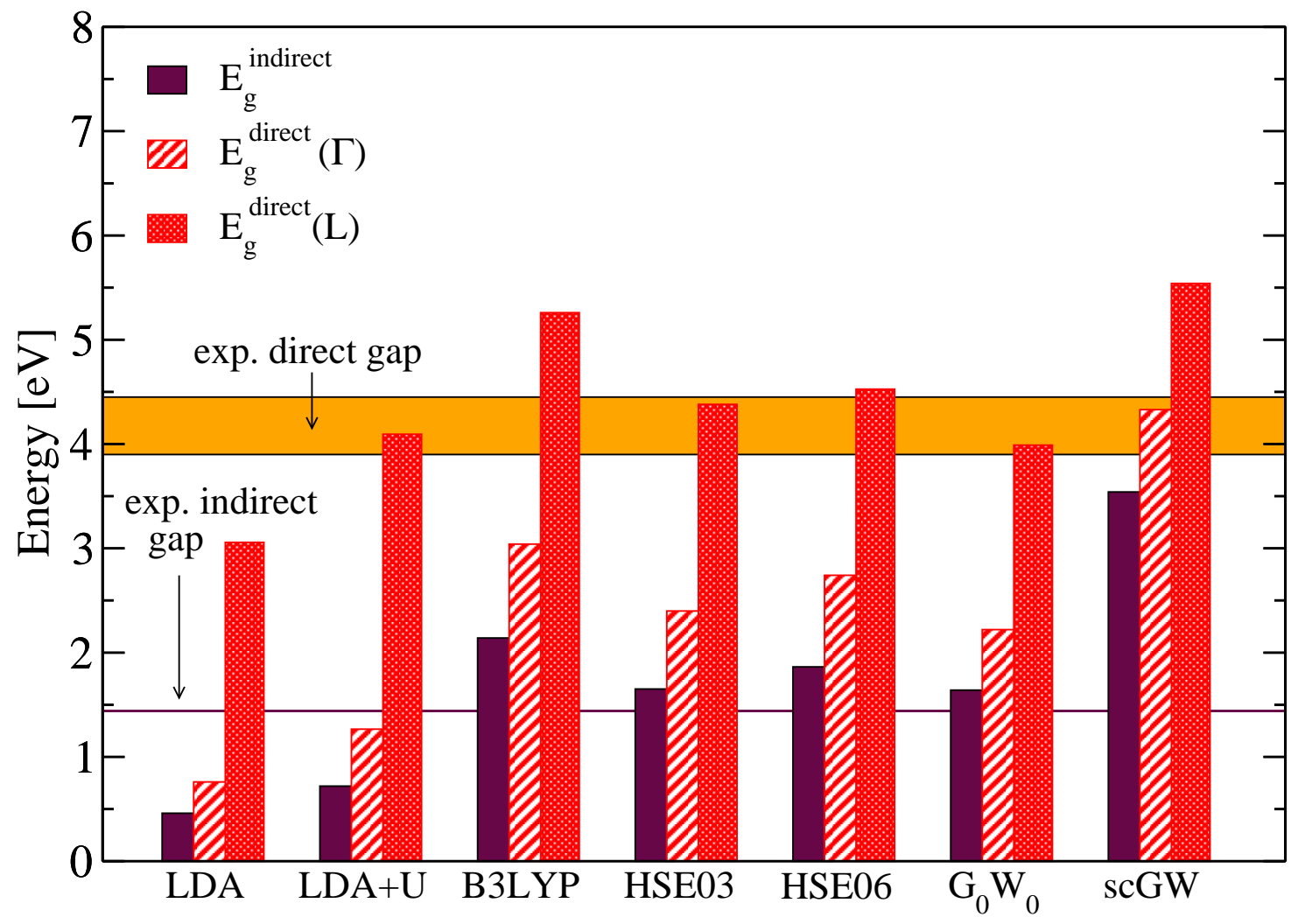

FIG. 3: (Color online) Band gaps of $\mathrm{CuInO}_{2}$ using: LDA, LDA $+U$, hybrid, $G_{0} W_{0}$, sc- $G W$. The horizontal zones contain data extracted from optical experiments (see text).

to $\mathrm{L}$ when sc- $G W$ is applied (see Fig. 2). Only $\mathrm{LDA}+U$ does not follow the trend, as it is the only case in which $E_{\mathrm{g}}^{\text {dir }}-E_{\mathrm{g}}^{\text {ind }}$ gets significantly larger than in LDA.

Looking at the direct gap, we point out that most of the methods give results that are within the experimental range, when an exciton binding energy of around $0.5 \mathrm{eV}$ [21] is considered. This is true for $\mathrm{LDA}+U, G_{0} W_{0}$, the hybrids HSE03 and HSE06. However, for sc- $G W$ and even for B3LYP, the theoretical gap is larger by about $1-1.5 \mathrm{eV}$ than the experimental findings. For $\mathrm{CuInO}_{2}$ (see Fig. 3) we have to make the comparison with care, as the smallest $E_{\mathrm{g}}^{\mathrm{dir}}$ is located at $\Gamma$, where optical transitions are forbidden [25]. A meaningful comparison with experiments must consider the gap at L. Thus, we find that both trends and quantitative results are analogous to those for $\mathrm{CuAlO}_{2}$. In particular, sc- $G W$ yields again $E_{\mathrm{g}}^{\mathrm{dir}}$ larger by $1-1.5 \mathrm{eV}$ than the experimental range.

We stress again that, to date, sc- $G W$ is arguably the best method available to estimate band gaps of wide-gap semiconductors, and that it gives excellent results for compounds like $\mathrm{Cu}_{2} \mathrm{O}$ and $\mathrm{CuIn}(\mathrm{S}, \mathrm{Se})_{2}[11,12]$. It is unlikely that the presence of defects can lead to such 
a large shrinkage of $E_{\mathrm{g}}^{\mathrm{dir}}$. However, there is another effect that has been neglected up to now: the change of screening due to the polarization of the lattice [31]. In fact, according to the experimental data [32], unfortunately available only for $\mathrm{CuAlO}_{2}$, the polaron constant for this system is large $\left(\alpha_{\mathrm{p}} \sim 1\right)$, indicating a non-negligible contribution of the lattice polarization to the electronic screening. In other ionic compounds with similar polaron constants this can lead to a shrinkage to the band gap by about $1 \mathrm{eV}$ [33]. A full sc- $G W$ calculation including in an ab initio framework the effects of the lattice polarization is to date beyond reach. However, a reliable estimate can be obtained using the model proposed by Bechstedt et al. [33], which gives a static representation of the polaronic effects based on difference of experimental static dielectric constants. By performing a perturbative $G W$ step including model polaronic effects on top of the sc-COHSEX, we found a uniform (kindependent) shrinkage of the band gap by $1.2 \mathrm{eV}$. As we can see in Fig. 1, this correction brings our results for $E_{\mathrm{g}}^{\text {dir }}$ well within the experimental range (once the excitonic correction of about $0.5 \mathrm{eV}$ is also considered). As it is observed in Ref. [33], the polaronic model employed can only overestimate the correction. All these results point to the conclusion that the agreement of the other methods with experiment was fortuitous and due to a cancellation of errors.

Looking now at the indirect gap, we focus on Fig. 1 as there are more experimental data for $\mathrm{CuAlO}_{2}$. All the hybrids and $G W$ calculations yield indirect gaps much larger than the experimental range $1.65-2.1 \mathrm{eV}$, even taking into account any possible excitonic and polaronic effects. Moreover, sc- $G W$, the best method used in this work, yields the highest $E_{\mathrm{g}}^{\text {ind }}$ at around $5 \mathrm{eV}$, while the difference $E_{\mathrm{g}}^{\mathrm{dir}}-E_{\mathrm{g}}^{\text {ind }}$ is in general much smaller than the experimental value $(\approx 2 \mathrm{eV})$, and even vanishing for the sc- $G W$ calculation. From Fig. 3 we realize that these conclusions are as well valid for $\mathrm{CuInO}_{2}$, where the best estimates for the indirect band gap is much larger than the experimental value of $1.44 \mathrm{eV}$ [10].

These are very strong arguments in favor of Robertson et al. [26] that suggested that the experimental "indirect gap" absorption was due to defects, and should not be present in the defect-free compound. Also Pellicer- Porres et al. [8] questioned the interpretation of the low energy peaks as indirect transitions, as the absorption coefficient is more than two orders of magnitude larger than in typical indirect absorption edges. The most promising defects are oxygen interstitials $\mathrm{O}_{i}$, as LDA calculations predict low formation energies and the introduction of states in the gap at 0.7 and $1.4 \mathrm{eV}$ [34]. However, a full clarification 
of this issue will require sc- $G W$ or hybrid calculations for these, and other more complex defects.

Finally, we analyze more in detail the band structures of $\mathrm{CuAlO}_{2}$ shown in Fig. 2. LDA calculations (red dashed lines) are compared with sc- $G W, \mathrm{HSE03}$, and LDA $+U$ calculations. The main effect of LDA $+U$ is to open the LDA gap by an amount that can be controlled by the parameter $U$. The difference $E_{\mathrm{g}}^{\mathrm{dir}}-E_{\mathrm{g}}^{\text {ind }}$ is in this approximation enhanced, due to a change of the character of the lowest conduction band along the symmetry lines. Hybrid calculations using HSE03 give a comparable $E_{\mathrm{g}}^{\mathrm{dir}}$ and a modified dispersion of both valence and conduction states close to the Fermi energy, which reduces $E_{\mathrm{g}}^{\mathrm{dir}}-E_{\mathrm{g}}^{\text {ind }}$. The conduction band minimum (CBM) within HSE03 is still located at $\Gamma$, but the difference between the $\mathrm{CBM}$ at $\mathrm{L}$ and $\Gamma$ gets significantly smaller. For sc- $G W$, besides the further increase of the band gaps, the dispersion of the bands is strongly affected by the many-body effects. In fact, the $G W$ corrections exhibit an unusual dispersion of around $1 \mathrm{eV}$ when looking at the different $\mathbf{k}$-points, displacing the CBM from $\Gamma$ to $\mathrm{L}$. We note that often in semiconductor physics one assumes that the quasiparticle corrections can be modeled by a rigid shift (the so-called scissor operator). From our results it follows that one should refrain from using this simple approximation for these important materials. We can also conclude that hybrid calculations give a better description of band dispersions than $\mathrm{LDA}+U$, even if the two approaches yield similar band gaps.

In conclusion, it is clear that the delafossite family exhibits complex and unusual band gap physics that can not be captured by standard theoretical approximations. We found that the direct band gap is well reproduced by the best many-body approaches if polaronic effects are taken into account. We can expect that this situation, of a large gap that is reduced substantially by polaronic effects, is quite general and is present in many more materials that previously expected. In fact, the apparent good agreement between calculated gaps (with hybrid functionals or $G_{0} W_{0}$ ) and experimental gaps for materials as simple and widely studied as LiF can be accidental, as preliminary calculations confirm: the underestimation of the gap by these methods (the sc $G W$ gap is indeed $2 \mathrm{eV}$ larger than the experimental and $G_{0} W_{0}$ gap) is compensated by the neglect of large polaronic effects. Furthermore, the modifications with respect to the LDA Kohn-Sham bands are strongly $\boldsymbol{k}$-dependent, which makes questionable the common practice of using a scissor operator. The band dispersion obtained by hybrid functional calculations is in between the LDA and sc- $G W$ dispersion, 
while the LDA $+U$ calculations open up the gap but do not give a significant improvement of the band dispersion. Finally, our calculations rule out the interpretation of the low energy features in the absorption spectra as arising from a putative indirect band gap. These structures should rather come from intrinsic defects, as proposed in Refs. [8, 26]. However, a complete understanding of the electronic and excitation properties of these systems will only be achieved, in our opinion, by a high-level theoretical scheme (like sc- $G W$ ) including defects and effects from the lattice polarization in an ab initio framework. Work along these lines is in progress.

We thank F. Bechstedt and A. Rubio for fruitful discussion. Part of the calculations were performed at the LCA of the University of Coimbra and at GENCI (project x2009096017). SB acknowledges funding from the European Community through the e-I3 ETSF project (Contract \#211956), MALM from the Portuguese FCT (PTDC/FIS/73578/2006) and from the French ANR (ANR-08-CEXC8-008-01), JV from an EDF/ANR CIFRE fellowship.

[1] S. Nakamura, M. Senoh, and N. Iwasa, Jpn. J. Appl. Phys. 31, L139 (1992); J. Neugebauer and C. G. Van de Walle, Phys. Rev. Lett. 75, 4452 (1995).

[2] F. Benko and F. Koffyberg, J. Phys. Chem. Solids 45, 57 (1984); H. Kawazoe et al., Nature 389, 939 (1997).

[3] K. Ueda et al., J. Appl. Phys. 89, 1790 (2001).

[4] H. Yanagi et al., Appl. Phys. Lett. 78, 1583 (2001).

[5] H. Yanagi et al., J. Appl. Phys. 88, 4159 (2000).

[6] C. Ong and H. Gong, Thin Solid Films 445, 299 (2003); T. Dittrich et al., Appl. Phys. Lett. 85, 742 (2004); E. Alkoy and P. Kelly, Vacuum 79, 221 (2005); A. Banerjee, R. Maity, and K. Chattopadhyay, Mat. Lett. 58, 10 (2004); A. Banerjee and K. Chattopadhyay, J. Appl. Phys. 97 (2005); A. Banerjee et al., Physica B 370, 264 (2005); R.-S. Yu et al., Appl. Phys. Lett. 90, 191117 (2007).

[7] S. Gilliland et al., Phys. Stat. Sol. (b) 244, 309 (2007).

[8] J. Pellicer-Porres et al., Appl. Phys. Lett. 88, 181904 (2006).

[9] C. W. Teplin et al., Appl. Phys. Lett. 85, 3789 (2004).

[10] M. Sasaki and M. Shimode, J. Phys. Chem. Solids 64, 1675 (2003). 
[11] F. Bruneval et al., Phys. Rev. Lett. 97, 267601 (2006).

[12] J. Vidal et al., Phys. Rev. Lett. (2010), accepted.

[13] E. N. Brothers et al., J. Chem. Phys. 129, 011102 (2008).

[14] J. Paier, M. Marsman, and G. Kresse, Phys. Rev. B 78, 121201 (2008).

[15] J. Hafner, J. Comput. Chem. 29, 2044 (2008).

[16] L. Hedin, Phys. Rev. 139, A796 (1965).

[17] W. G. Aulbur, L. Jönsson, and J. Wilkins, Solid State Phys. 54, 1 (2000).

[18] M. Gatti et al., Phys. Rev. Lett. 99, 266402 (2007).

[19] S. V. Faleev, M. van Schilfgaarde, and T. Kotani, Phys. Rev. Lett. 93, 126406 (2004); M. van Schilfgaarde, T. Kotani, and S. Faleev, Phys. Rev. Lett. 96, 226402 (2006).

[20] F. Bruneval, N. Vast, and L. Reining, Phys. Rev. B 74, 045102 (2006).

[21] R. Laskowski et al., Phys. Rev. B 79, 165209 (2006).

[22] P. Stephens et al., J. Phys. Chem. 98, 11623 (1994).

[23] J. Heyd, G. E. Scuseria, and M. Ernzerhof, J. Chem. Phys. 118, 8207 (2003); J. Heyd, G. E. Scuseria, and M. Ernzerhof, J. Chem. Phys. 124, 219906 (2006).

[24] B. J. Ingram et al., Phys. Rev. B 64, 155114 (2001); L. Shi, Z. Fang, and J. Li, J. Appl. Phys. 104, 073527 (2008).

[25] X. Nie, S. Wei, and S. Zhang, Phys. Rev. Lett. 88, 066405 (2002).

[26] J. Robertson et al., Thin Solid Films 411, 96 (2002).

[27] M. N. Huda et al., Phys. Rev. B 80, 035205 (2009).

[28] G. Kresse and J. Furthmüller, Comput. Mater. Sci. 6, 15 (1996); G. Kresse and J. Furthmüller, Phys. Rev. B 54, 11169 (1996).

[29] X. Gonze et al., Z. Kristallogr. 220, 558 (2005).

[30] F. Bruneval and X. Gonze, Phys. Rev. B 78, 085125 (2008).

[31] W. B. Fowler, Phys. Rev. 151, 657 (1966); A. B. Kunz, Phys. Rev. B 6, 606 (1972).

[32] J. Pellicer-Porres, A. Segura, and D. Kim, Semicond. Sci. Technol. 24, 015002 (2009).

[33] F. Bechstedt et al., Phys. Rev. B 72, 245114 (2005).

[34] I. Hamada and H. Katayama-Yoshida, Physica B 376-377, 808 (2006). 